\title{
Effect of salt bridges rupture on the activity and thermostability of bovine chymosin
}

\author{
E.S. Novoselova ${ }^{1 *}$, A.P. Rudometov ${ }^{2}$, A.V. Kriger ${ }^{3}$, V.V. Elchaninov ${ }^{3}$ \\ ${ }^{1}$ Novosibirsk State University, Novosibirsk, Russia \\ ${ }^{2}$ State Research Center of Virology and Biotechnology "Vector" Rospotrebnadzor, Koltsovo, Russia \\ ${ }^{3}$ Federal Altai Scientific Centre of Agro-BioTechnologies, Barnaul, Russia \\ *e-mail: batmaiden@gmail.com
}

Key words: salt bridges, chymosin, thermostability, molecular dynamics

\begin{abstract}
Motivation and Aim: It is known that salt bridges are one of the factors influencing the thermostability of bovine chymosin [1]. Salt bridges result from the interaction of positively and negatively charged side radicals of amino acid residues on the surface of the protein. This work aimed at creating the functional mutants of chymosin with reduced thermostability. Chymosin is an enzyme that catalyzes the hydrolytic cleavage of the peptide bond between phenylalanine and methionine of the main protein of kappacasein milk with the formation of a milk clot.
\end{abstract}

Methods and Algorithms: To determine salt bridges, molecular dynamics in the GROMACS 5 program at various temperatures - 300, 350, and $400 \mathrm{~K}$ was used. The trajectories of $5 \mathrm{~ns}$ duration were obtained for each temperature. The program VMD 1.9.3 was used for processing the trajectories. Mutant variants of chymosin were obtained using site-directed mutagenesis. The initial and mutant variants of chymosin were obtained using the E. coli pET21a vector system. The activity of the enzyme was determined by measuring the milk-coagulating activity on a standardized dry milk substrate. Thermostability was determined by the change in enzyme activity with increasing temperature.

Results: 10 salt bridges with persistence of no less than $50 \%$ were detected in chymosin at least one of the investigated temperatures. Four of them were selected for the destruction effect to be investigated experimentally.

For the two mutant variants E198K and E363Q, the milk-binding activity was too weak to be further investigated. For the other two mutant variants D156V and D209N, the milk-binding activity was lower than that of the wild-type, but it was noticeable. Thermostability of these two variants proved to be higher than that of the wild-type chymosin.

Conclusion: The mutant variants of chymosin with disrupted salt bridges were investigated. Such point mutations turned out to significantly affect the milk-swilling activity. Surprisingly, an increase in thermal stability was obtained in two cases instead of the expected reduction in thermal stability. These results demonstrate how great could be the effect of a single mutation on the enzyme activity and it can encourage the further research.

Acknowledgements: The reported study was funded by RFBR according to the research project No. 17-44-220540 Supported by Russian Ministry of Science and Education under 5-100 Excellence Programme.

\section{References}

1. Karshikoff et al. (2015) Rigidity versus flexibility: the dilemma of understanding protein thermal stability. FEBS J. 282(20):3899-917. 